\title{
3D Printed Models In Pregnancy And Its Utility In Improving Psychological Constructs: A Case Series
}

John Joseph Coté ( $\nabla$ john.cote@commonspirit.org )

Creighton University Medical Center

Brayden Patric Coté

Creighton University

Amy Badura-Brack

Creighton University

\section{Case Report}

Keywords: 3D printing, maternal-fetal attachment, paternal-fetal attachment, anxiety, depression, twins, facial cleft

Posted Date: March 3rd, 2022

DOI: https://doi.org/10.21203/rs.3.rs-1409940/v1

License: (c) (1) This work is licensed under a Creative Commons Attribution 4.0 International License. Read Full License 


\section{Abstract}

Background: Additive manufacturing is being utilized in almost every aspect of medicine. 3D printing has especially been used in conjunction with 3D ultrasonography to assist in antenatal assessment and presurgical planning with fetal malformations. As printing capabilities improve and applications are explored there may be more advantages for all parents to visualize and touch 3D printed models of their fetus.

Case Presentation: We present three cases involving 3D printed models and four different but interrelated psychological constructs- antenatal depression, antenatal anxiety, maternal-fetal attachment, and paternal-fetal attachment. Each case shows for the first time possible beneficial effects within these prevalent and significant problems.

Conclusions: The degree to which the anxiety, depression, and attachment scores improved after the presentation of the 3D printed models is encouraging. Randomized controlled trials utilizing 3D printed models to improve psychological constructs should be supported considering the findings within these four cases.

\section{Introduction}

Maternal-fetal attachment (MFA), paternal-fetal attachment (PFA), perinatal anxiety and depression are psychological constructs facing pregnant couples today. While the prevalence of maternal antenatal depression (20.7\% 95\% Cl 19.4-21.9 [1]) and maternal anxiety (24.6\% 95\% $\mathrm{Cl} 21.2-28.0$ [2]) is well documented, MFA and PFA have been studied less frequently. The list of negative impacts on pregnant women, their fetuses and infants related to antenatal depression, anxiety and low MFA is staggering. These psychological factors are associated with placental abruption, gestational diabetes, preeclampsia/hypertension, preterm birth, intra-uterine fetal demise, and maternal death[3-13]. Pharmacologic therapies have been utilized to treat depression and anxiety successfully but their safety in pregnancy has been debated[14] and treatment with pharmacologic or non-pharmacologic interventions have not been proven to reduce the health risks commonly associated with antenatal depression or anxiety beyond psychological symptoms[15]. MFA and PFA have received even less clinical attention and research aimed at designing interventions to improve attachment scores in pregnancy $[16$, 17]. Recently a study showed that offering a third-trimester routine ultrasound does not have a psychological benefit for all pregnant women, yet it might improve MFA for women with higher levels of depressive symptoms[18]. A review also found that an MFA increase using various interventions can reduce induced anxiety and depression[19]. Studies have shown that fetal ultrasounds can improve MFA and while individual studies have not shown the utility of 3D ultrasonography over 2D ultrasonography, the trend favors the more accurate representation of the fetus[20-22].

3D printing technology, especially additive manufacturing has been reviewed for its utility in gynecology in a variety of instances: fibroids, endometriosis, pelvic floor disorders, gynecological oncology, patient 
education for surgical procedures, student education, and simulation-based training[23]. In obstetrics 3D printing technology has been used in cases of placenta accreta, fetal congenital anomalies for presurgical planning, and interactions with visually impaired pregnant patients[24-27]. Although we are unaware of any studies examining the impact of 3D printed technology on antenatal depression or anxiety, a few studies have shown significant improvement in MFA with 3D printed fetal models[27-29].

This report describes a series of three cases examining the use of 3D printed models' utility in improving MFA, PFA, antenatal depression and anxiety.

\section{Cases}

\section{Case One}

A 35-year-old G3P1011 presented to the clinic for prenatal care with her 35-year-old husband who had fathered all her pregnancies and no others. An ultrasound performed at her initial visit showed a viable diamniotic/dichorionic twin pregnancy. Genetic amniocentesis and NIPT were declined. The patient and her husband were both healthy with no abnormal past medical or past surgical history. The patient had only been taking her prenatal vitamin and was started on a baby aspirin $81 \mathrm{mg}$. The patient and her husband provided consent for the case report, in accordance with Creighton Institutional Review Board (IRB) standards at 15 weeks. The patients were told that she would need to answer the maternal antenatal attachment scale (MAAS) questionnaire and he would need to answer the paternal antenatal attachment scale (PAAS)[30, 31] at four different timepoints. Additionally, both parents would 'journal their thoughts, feelings or emotions about the pregnancy and/or twins'. The study included assessments before and after each of the two study ultrasounds and subsequent 3D printed models. The first preassessment followed by the first ultrasound was obtained during the second trimester at 15 weeks. The second pre-assessment followed by the second ultrasound was obtained during the third trimester at 28 weeks. Both participants watched the screening in real-time. The GE Voluson ${ }^{\mathrm{TM}} \mathrm{E} 10$ exported the Digital Imaging and Communications in Medicine (DICOM) information and this was transferred to 3D slicer[32]. 3D Slicer segmentation of the volumetric data was performed, and the stereolithography (STL) file was loaded to the Cura Lulzbot ${ }^{\circledR}$ program. The Lulzbot TAZ Workhorse ${ }^{\circledR} 3 \mathrm{D}$ printer was used to print a model of both fetal faces together using polylactic acid (PLA) (Figure 1A).

Figure $1 \mathrm{~A}$. Timing of questionnaires and interventions throughout the pregnancy

When the study concluded, both journals were transcribed separately into the IBM Watson ${ }^{\mathrm{TM}}$ Knowledge Studio on IBM Cloud® to perform sentiment and emotional analysis[33].

The MAAS scores were $85,87,90,90$ and the PAAS scores were $52,55,59,65$. Analyzing the journals revealed positive content for both parents. The maternal sentiment score was 0.63 and the paternal sentiment score was 0.53 on a scale of -1.00 (negative) to +1.00 (positive). Emotional analysis scores range from 0 to 1 to indicate the presence of the emotion. Emotional analysis of the entire maternal journal resulted in emotional scores of 0.64 'joy', 0.60 'fear', 0.56 'sadness', 0.07 'anger', and 0.05 'disgust'. 
Emotional analysis of the paternal journal revealed similarly high levels of 0.67 'joy' and 0.57 'fear', lower levels of 0.16 'sadness', and similar negligible levels of 0.05 'anger' and 0.03 'disgust'. Sample maternal diary entries mentioning the 3D printed models are listed below.

Today we got our 3-D print of the twins! Wow! This was so neat! We showed it to our family and sent pictures to some friends and everyone was in awe. I set the shadow box on the dresser in our bedroom so both [my husband] and I get to see it every day. This made me so happy and again, just helps to make it that much more real that we are actually having TWINS!

The sentiment appeared very positive at 0.85 . Emotional analysis 0.61 'joy', 0.07 'fear', 0.11 'sadness', 0.02 'disgust' and 0.04 'anger' paralleled the high sentiment.

Today was such a special day. [My toddler] has been taking notice of the 3-D shadow box of the twins on my dresser. For the past several days, he points to it and says "bebies"... . today, [he] kept pointing to the box and wanted to hold it. We let him look at it and explore a bit. [My toddler] again pointed to the glass and said "bebies", kisses. It was the sweetest thing l've ever seen.

The sentiment again was positive at 0.44 . Emotional analysis was 0.56 'joy', 0.07 'fear', 0.54 'sadness', 0.06 'disgust', and 0.08 'anger'

\section{Case Two}

A 21-year-old G3P0110 presented to clinic for prenatal care. An ultrasound performed at the initial visit showed a viable fetus at 8 weeks and 4 days. A 3D image was obtained at her early pregnancy ultrasound. Her history was significant for a 24-week delivery, due to severe pre-eclampsia, by cesarean section. The baby passed away on day of life 41 due to prematurity. She also had a previous early miscarriage at 9 weeks and 4 days. She had been taking Xanax ${ }^{\circledR}$ prior to the pregnancy for anxiety but had stopped after she had discovered she was pregnant. The patient provided consent for the case report, in accordance with Creighton IRB standards, at 12 weeks and 4 days. She was told she would need to answer a generalized anxiety disorder questionnaire (GAD-7) [34] and patient health questionnaire (PHQ9) [35], at two time points. After consenting to the study and answering the questionnaires a 3D printed model of her 8-week-old fetus was presented to the patient (Figure 1B).

Figure 1B. Eight-week-old fetus

Taking the DICOM data from the 8-week ultrasound (GE Voluson ${ }^{\mathrm{TM}} \mathrm{E8}$ ) and converting it to STL created the image. The segmented image was printed on an Ultramaker 2+® 3D printer using PLA[36]. One week after the presentation of 3D printed model, the patient answered the GAD-7 and PHQ-9 again.

The GAD-7 initial score was 13, moderately severe anxiety disorder. The answer to question number 8 , "If you checked off any problems, how difficult have these problems made it for you to do your work, take care of things at home, or get along with other people" was "very difficult". The PHQ-9 initial score was 19 , moderately severe depression. Both anxiety and depression scores improved after the presentation of 
the 3D printed model. The GAD-7 score decreased to 6, mild anxiety disorder. The answer to the question number 8 changed to "somewhat difficult". The PHQ-9 decreased to 7, mild depression.

\section{Case Three}

A 36-year-old G1P0 presented to clinic for prenatal care. An ultrasound performed at the 20-week fetal anatomic survey showed a fetus that had a suspected unilateral cleft lip and palate. Genetic amniocentesis and Non-Invasive Prenatal Testing (NIPT) were declined. No other anomalies were detected on follow-up ultrasounds. Patient had been taking Zoloft ${ }^{\circledR}$ during the pregnancy for depression. The patient provided consent for the case report, in accordance with Creighton IRB standards, at 30 weeks. She was told she would need to answer a PHQ-9 questionnaire and a MAAS questionnaire initially, and then again in two weeks. After consenting her, she completed the PHQ-9 and MAAS questionnaires. An ultrasonographer, who had neutral interactions, performed a 20-minute 3D/4D ultrasound (GE Voluson $^{\mathrm{TM}} \mathrm{E} 10$ ) and attempted to specifically target the fetus' face. The patient was able to watch the screening in real-time on a large screen television. After the patient left the clinic, the DICOM data from the 3D ultrasound was extracted, segmented, cleaned, and converted to an STL file. The image was printed by an Ultimaker $2+\circledR 3 D$-printer using PLA. One week after the initial ultrasound, the patient was asked to return to the clinic to receive a small shadow box containing the 3D printed model of her baby's face produced from the ultrasound data (Figure 1C).

Finally, two weeks after the study ultrasound the patient completed the PHQ-9 and MAAS questionnaires again by phone.

The PHQ-9 initial score was 7, mild depression. The MAAS initial score was 65, with the time in attachment at 23 and the quality of attachment at 39. One week after the presentation of the 3D printed model the PHQ-9 score decreased to 4, minimal depression and the MAAS score increased to 77, with the time in attachment increasing to 27 and the quality of attachment increasing to 45.

\section{Discussion And Conclusions}

There are established links between a loss in a previous pregnancy and the current pregnancy with regards to anxiety, depression, and MFA [37,38]. There is also evidence that 3D printed models may improve MFA, but this is the first time 3D printed models have been shown to potentially improve anxiety, depression and PFA within a pregnancy. Looking at depression, anxiety, and attachment through the lens of the 3D print as a type of non-pharmacologic treatment opens a vast array of applications for this technology. While researchers will look to transformative applications such as organ fabrication, customized prosthetics, and drug dosage and delivery applications for 3D printing [39] these are examples of current real time applications for ultrasounds and 3D printing that every obstetrician can begin to explore. A robust expanded cohort should be examined to determine if 3D printing of early pregnancies would help improve outcomes for women in pregnancies following a fetal or neonatal loss, and if 3D printed models can increase MFA and/or PFA in pregnancies, which include fetal facial clefts and twins. 


\section{Abbreviations}

MFA: maternal-fetal attachment; PFA: paternal-fetal attachment; MAAS: maternal antenatal attachment scale; PAAS: paternal antenatal attachment scale; GAD: generalized anxiety disorder; PHQ: patient health questionnaire; NIPT: non-invasive prenatal testing; PLA: polylactic acid; DICOM: digital imaging and communications in medicine; IRB: institutional review board; $\mathrm{Cl}$ : confidence interval; 3D: three dimensional; 4D: four dimensional; GE: general electric; STL: stereolithography; IBM: international business machines.

\section{Declarations}

Ethics approval and consent to participate

Creighton IRB exempt. All participants have written consents for participation and publication. Consents are available upon request.

Availability of data and materials

All data generated or analyzed during the current study are available from the corresponding author upon reasonable request.

\section{Competing interests}

$\mathrm{JC}$ and $\mathrm{BC}$ disclose a competing interest as they have connections with a 3D printing company. $\mathrm{ABB}$ has no competing interest.

\section{Funding}

No funding

\section{\&Authors' contributions}

$\mathrm{JC}$ designed the study, interpreted the patient data, wrote, and revised the manuscript. ABB interpreted the patient data wrote and revised the manuscript. BC wrote and revised the manuscript. All authors read and approved the final manuscript.

\section{Acknowledgements}

While not meeting the requirements for authorship, we would like to acknowledge Joseph Haggerty MD and Nicholas Dubay MD who presented parts of this research at the Construct3D Conference 2020 at Rice University in Houston, TX. Consent for acknowledgment was obtained.

\section{References}


[1] X. Yin et al., "Prevalence and associated factors of antenatal depression: Systematic reviews and meta-analyses," Clinical psychology review, p. 101932, 2020. https://doi.org/10.1016/j.cpr.2020.101932

[2] C.-L. Dennis, K. Falah-Hassani, and R. Shiri, "Prevalence of antenatal and postnatal anxiety: systematic review and meta-analysis," The British Journal of Psychiatry, vol. 210, no. 5, pp. 315-323, 2017. https://doi.org/10.1192/bjp.bp.116.187179

[3] N. C. de Paz et al., "Risk of placental abruption in relation to maternal depressive, anxiety and stress symptoms," Journal of affective disorders, vol. 130, no. 1-2, pp. 280-284, 2011. https://doi.org/10.1016/j.jad.2010.07.024

[4] C. Minschart et al., "Antenatal Depression and Risk of Gestational Diabetes, Adverse Pregnancy Outcomes, and Postpartum Quality of Life," The Journal of Clinical Endocrinology \& Metabolism, 2021. https://doi.org/10.1210/clinem/dgab156

[5] T. K. Chung, T. K. Lau, A. S. Yip, H. F. Chiu, and D. T. Lee, "Antepartum depressive symptomatology is associated with adverse obstetric and neonatal outcomes," Psychosomatic medicine, vol. 63, no. 5, pp. 830-834, 2001. https://doi.org/10.1097/00006842-200109000-00017

[6] B. A. Dachew, J. G. Scott, and R. Alati, "Hypertensive disorders during pregnancy and perinatal mental health symptoms," Journal of Affective Disorders Reports, p. 100245, 2021. https://doi.org/10.1016/j.jadr.2021.100245

[7] R. Hu, Y. Li, Z. Zhang, and W. Yan, "Antenatal depressive symptoms and the risk of preeclampsia or operative deliveries: a meta-analysis," PloS one, vol. 10, no. 3, p. e0119018, 2015.

https://doi.org/10.1371/journal.pone.0119018

[8] S. E. Sanchez et al., "Risk of spontaneous preterm birth in relation to maternal depressive, anxiety and stress symptoms," The Journal of reproductive medicine, vol. 58, p. 25, 2013. PMCID: PMC3662498

[9] S. D. Simonovich et al., "Meta-Analysis Of Antenatal Depression And Adverse Birth Outcomes In US Populations, 2010-20: Study is a meta-analysis of antenatal depression and adverse birth outcomes in the US, 2010-20," Health Affairs, vol. 40, no. 10, pp. 1560-1565, 2021.

https://doi.org/10.1377/hlthaff.2021.00801

[10] A. G. Khanghah, Z. B. Khalesi, and R. H. Afagh, "The importance of depression during pregnancy," JBRA assisted reproduction, vol. 24, no. 4, p. 405, 2020. https://doi.org/10.5935/1518-0557.20200010

[11] P. Bansil et al., "Maternal and fetal outcomes among women with depression," Journal of Women's Health, vol. 19, no. 2, pp. 329-334, 2010. https://doi.org/10.1089/jwh.2009.1387

[12] K. Mangla, M. C. Hoffman, C. Trumpff, S. O'Grady, and C. Monk, "Maternal self-harm deaths: an unrecognized and preventable outcome," American journal of obstetrics and gynecology, vol. 221, no. 4, 
pp. 295-303, 2019. https://doi.org/10.1016/j.ajog.2019.02.056

[13] L. Rossen et al., "Predictors of postnatal mother-infant bonding: the role of antenatal bonding, maternal substance use and mental health," Archives of women's mental health, vol. 19, no. 4, pp. 609622, 2016. https://doi.org/10.1007/s00737-016-0602-z

[14] M. Dubovicky, K. Belovicova, K. Csatlosova, and E. Bogi, "Risks of using SSRI/SNRI antidepressants during pregnancy and lactation," Interdisciplinary toxicology, vol. 10, no. 1, p. 30, 2017. https://doi.org/ 10.1515/intox-2017-0004

[15] A. Jarde et al., "Does non-pharmacological therapy for antenatal depression reduce risks for the infant?," Archives of women's mental health, vol. 19, no. 3, pp. 549-552, 2016. https://doi.org/10.1007/s00737-015-0577-1

[16] B. L. Cannella, "Maternal-fetal attachment: an integrative review," Journal of Advanced Nursing, vol. 50, no. 1, pp. 60-68, Apr 2005. https://doi.org/10.1111/j.1365-2648.2004.03349.x

[17] E. Abasi, A. Keramat, N. S. Borghei, S. Goli, and M. Farjamfar, "Evaluating the effect of prenatal interventions on maternal-foetal attachment: A systematic review and meta-analysis," Nursing Open, vol. 8, no. 1, pp. 4-16, Jan 2021. https://doi.org/10.1002/nop2.648

[18] M. Westerneng et al., "The effect of offering a third-trimester routine ultrasound on pregnancy-specific anxiety and mother-to-infant bonding in low-risk women: A pragmatic cluster-randomized controlled trial," Birth, Jul 20 2021. https://doi.org/10.1111/birt.12573

[19] P. Mahmoudi, F. Elyasi, A. Nadi, and M. A. Shirvani, "Effect of maternal-fetal/Neonatal attachment interventions on perinatal anxiety and depression: A narrative review," Journal of Nursing and Midwifery Sciences, vol. 7, no. 2, p. 126, 2020. https://doi.org/ 10.4103/JNMS.JNMS_28_19

[20] E. A. de Jong-Pleij, L. S. Ribbert, L. R. Pistorius, E. Tromp, E. J. Mulder, and C. M. Bilardo, "Threedimensional ultrasound and maternal bonding, a third trimester study and a review," Prenat Diagn, vol. 33, no. 1, pp. 81-8, Jan 2013. https://doi.org/10.1002/pd.4013

[21] B. Sedgmen, C. McMahon, D. Cairns, R. J. Benzie, and R. L. Woodfield, "The impact of twodimensional versus three-dimensional ultrasound exposure on maternal-fetal attachment and maternal health behavior in pregnancy," Ultrasound in Obstetrics \& Gynecology, vol. 27, no. 3, pp. 245-251, Mar 2006. https://doi.org/10.1002/uog.2703

[22] M. Rustico, C. Mastromatteo, M. Grigio, C. Maggioni, D. Gregori, and U. Nicolini, "Two-dimensional vs. two-plus four-dimensional ultrasound in pregnancy and the effect on maternal emotional status: a randomized study," Ultrasound in obstetrics \& gynecology, vol. 25, no. 5, pp. 468-472, May 2005. https://doi.org/10.1002/uog.1894 
[23] T. E. Flaxman, C. M. Cooke, O. X. Miguel, A. M. Sheikh, and S. S. Singh, "A review and guide to creating patient specific 3D printed anatomical models from MRI for benign gynecologic surgery," 3D Printing in Medicine, vol. 7, no. 1, pp. 1-10, 2021. https://doi.org/10.1186/s41205-021-00107-7

[24] J. Liang et al., "Feasibility Analysis of 3D Printing With Prenatal Ultrasound for the Diagnosis of Fetal Abnormalities," Journal of Ultrasound in Medicine, 2021. https://doi.org/10.1002/jum.15821

[25] J. Yang, X. Pan, W. Pan, M. Yang, J. Liu, and J. Tang, "3D Printing of Congenital and Prenatal Heart Diseases," in Cardiovascular 3D Printing: Springer, 2021, pp. 35-52. https://doi.org/10.1007/978-981-156957-9_5

[26] R. Nicot, E. Hurteloup, S. Joachim, C. Druelle, and J.-M. Levaillant, "Using low-cost 3D-printed models of prenatal ultrasonography for visually-impaired expectant persons," Patient Education and Counseling, 2021. https://doi.org/10.1016/j.pec.2021.02.033

[27] H. Werner, J. Lopes, G. Tonni, and E. Araujo Junior, "Maternal-fetal attachment in blind women using physical model from three-dimensional ultrasound and magnetic resonance scan data: six serious cases," J Matern Fetal Neonatal Med, vol. 29, no. 14, pp. 2229-32, 2016.

https://doi.org/10.3109/14767058.2015.1085015

[28] J. J. Coté, B. Thomas, and J. Marvin, "Improved maternal bonding with the use of 3D-printed models in the setting of a facial cleft," Journal of 3D Printing in Medicine, vol. 2, no. 3, pp. 97-102, 2018. https://doi.org/10.2217/3dp-2018-0005

[29] J. J. Coté, A. S. Badura-Brack, R. W. Walters, N. G. Dubay, and M. R. Bredehoeft, "Randomized Controlled Trial of the Effects of 3D-Printed Models and 3D Ultrasonography on Maternal-Fetal Attachment," J Obstet Gynecol Neonatal Nurs, vol. 49, no. 2, pp. 190-199, Mar 2020. https://doi.org/10.1016/j.jogn.2020.01.003

[30] J. T. Condon, "The assessment of antenatal emotional attachment: development of a questionnaire instrument," Br J Med Psychol, vol. 66 ( Pt 2), pp. 167-83, Jun 1993. https://doi.org/10.1111/j.20448341.1993.tb01739.x

[31] J. T. Condon, "The Parental-foetal relationship-a comparison of male and female expectant parents," Journal of Psychosomatic Obstetrics \& Gynecology, vol. 4, no. 4, pp. 271-284, 1985. https://doi.org/10.3109/01674828509016729

[32] R. Kikinis, S. D. Pieper, and K. G. Vosburgh, "3D Slicer: a platform for subject-specific image analysis, visualization, and clinical support," in Intraoperative imaging and image-guided therapy. Springer, 2014, pp. 277-289. https://doi.org/10.1007/978-1-4614-7657-3_19

[33] S. B. Goldberg et al., "Machine learning and natural language processing in psychotherapy research: Alliance as example use case," Journal of counseling psychology, vol. 67, no. 4, p. 438, 2020. 
[34] R. L. Spitzer, K. Kroenke, J. B. Williams, and B. Löwe, "A brief measure for assessing generalized anxiety disorder: the GAD-7," Archives of internal medicine, vol. 166, no. 10, pp. 1092-1097, May 222006. https://doi.org/ 10.1001/archinte.166.10.1092

[35] K. Kroenke, R. L. Spitzer, J. B. Williams, and B. Löwe, "The patient health questionnaire somatic, anxiety, and depressive symptom scales: a systematic review," General hospital psychiatry, vol. 32, no. 4, pp. 345-359, Jul-Aug 2010. https://doi.org/10.1016/j.genhosppsych.2010.03.006

[36] B. Tutschek, "3D prints from ultrasound volumes," Ultrasound in Obstetrics \& Gynecology, Aug 27 2018. https://doi.org/10.1002/uog.20108

[37] C. Gaudet, N. Séjourné, L. Camborieux, R. Rogers, and H. Chabrol, "Pregnancy after perinatal loss: Association of grief, anxiety and attachment," Journal of Reproductive and Infant Psychology, vol. 28, no. 3, pp. 240-251, 2010. https://doi.org/10.1080/02646830903487342

[38] L. Lee, K. McKenzie-McHarg, and A. Horsch, "The impact of miscarriage and stillbirth on maternalfetal relationships: an integrative review," J Reprod Infant Psychol, vol. 35, no. 1, pp. 32-52, Feb 2017. https://doi.org/10.1080/02646838.2016.1239249

[39] C. L. Ventola, "Medical applications for 3D printing: current and projected uses," Pharmacy and Therapeutics, vol. 39, no. 10, p. 704, 2014. PMID: 25336867

\section{Figures}

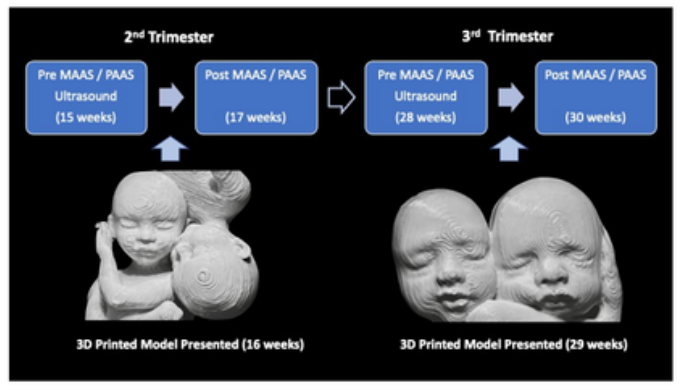

(A)

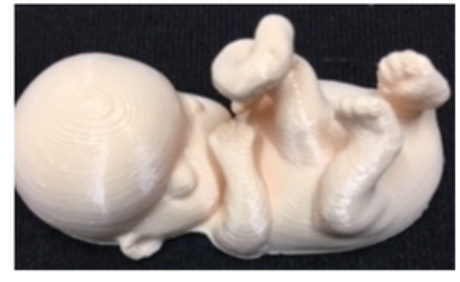

(B)

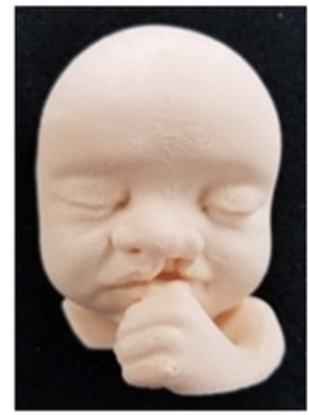

(C)

\section{Figure 1}
A. Timing of questionnaires and interventions throughout the pregnancy B. Eight-week-old fetus
C. Fetus with facial cleft at 30-weeks 